\title{
RELATIVISTIC EFFECTS IN ELECTROWEAK NUCLEAR RESPONSES
}

\author{
MARIA B. BARBARO \\ Dipartimento di Fisica Teorica, Università di Torino, and INFN, Sezione di \\ Torino, Via Giuria 1, 10128 Torino, Italy \\ E-mail: barbaro@to.infn.it
}

\begin{abstract}
The electroweak response functions for inclusive electron scattering are calculated in the Relativistic Fermi Gas model, both in the quasi-elastic and in the $\Delta$ peak regions. The impact of relativistic kinematics at high momentum transfer is investigated through an expansion in the initial nucleonic momentum, which is however exact in the four-momentum of the exchanged boson. The same expansion is applied to the meson exchange currents in the particle-hole sector: it is shown that the non-relativistic currents can be corrected by simple kinematical factors to account for relativity. The left-right asymmetry measured via polarized electron scattering is finally evaluated in the quasi-elastic and $\Delta$ peaks.
\end{abstract}

\section{Introduction}

The possibility of extracting from polarized electron scattering on protons the single nucleon strange and axial form factors has been the focus of many recent studies. Also the electroexcitation of the $\Delta$ provides important informations on the very poorly known $N \rightarrow \Delta$ transition form factors. However the scattering on a free proton is insufficient to disentangle all the form factors involved in the process: hence the opportunity to perform experiments on complex nuclei has been suggested $\mathbb{t}$.

Since the double differential asymmetry for inclusive electron scattering

$$
\mathcal{A}=\frac{d^{2} \sigma(h=+1)-d^{2} \sigma(h=-1)}{d^{2} \sigma(h=+1)+d^{2} \sigma(h=-1)}
$$

( $h$ being the helicity of the initial electron) is an extremely small quantity - roughly of the order of $10^{-5}$ - whose absolute value grows with $\left|Q^{2}\right|$, a relativistic treatment of the reaction in both the quasi-elastic (QE) and $\Delta$ peak is needed.

The most appropriate theoretical framework for a consistent study of relativistic effects is the Relativistic Fermi Gas (RFG) model, which, in spite of its simplicity, is capable of well describing the qualitative features of both peaks, fulfilling the fundamental requirements of Lorentz covariance and gauge invariance. Moreover for a non-interacting system the RFG yields analytical 
expressions for the response functions, allowing for a clear physical interpretation of each contribution. On the basis of free RFG nuclear correlations can then be treated perturbatively, still preserving the two above mentioned properties.

\section{Relativistic Fermi Gas response functions}

The asymmetry (11) can be expressed in terms of 5 inclusive response functions, the longitudinal and transverse electromagnetic $R^{L}$ and $R^{T}$ and the longitudinal, transverse and axial $\widetilde{R}^{L}, \widetilde{R}^{T}$ and $\widetilde{R}^{T^{\prime}}$ parity-violating ones, associated to the interference between the photon and $Z^{0}$ exchange, according to

$$
\mathcal{A}=\mathcal{A}_{0} \frac{v_{L} \widetilde{R}^{L}+v_{T} \widetilde{R}^{T}+v_{T^{\prime}} \widetilde{R}^{T^{\prime}}}{v_{L} R^{L}+v_{T} R^{T}}
$$

$v_{L, T, T^{\prime}}$ being kinematical factors and $\mathcal{A}_{0} \approx 3.1 \times 10^{-4} \tau$.

In the RFG model the nucleus is treated as a collection of free nucleons described by positive energy Dirac spinors with $E(p) \overline{\overline{\bar{F}}} \sqrt{p^{2}+m_{N}^{2}}$ : the above responses are then analytic and factorize as follows

$$
\begin{aligned}
R^{L, T}(\kappa, \lambda) & =\frac{3 \mathcal{N} \xi_{F}}{4 \kappa m_{N} \eta_{F}^{3}}\left[1-\psi^{2}(\kappa, \lambda)\right] U^{L, T}(\kappa, \lambda) \\
\widetilde{R}^{L, T, T^{\prime}}(\kappa, \lambda) & =\frac{3 \mathcal{N} \xi_{F}}{4 \kappa m_{N} \eta_{F}^{3}}\left[1-\psi^{2}(\kappa, \lambda)\right] \widetilde{U}^{L, T, T^{\prime}}(\kappa, \lambda),
\end{aligned}
$$

where $\mathcal{N}$ is the number of protons or neutrons, $\kappa=q / 2 m_{N}$ and $\lambda=\omega / 2 m_{N}$ are the dimensionless momentum and energy transfers, $\eta_{F}=p_{F} / m_{N}$ and $\xi_{F}=\sqrt{\eta_{F}^{2}+1}-1$ the Fermi momentum and kinetic energy and

$$
\psi^{2}(\kappa, \lambda)=\frac{\epsilon_{0}-1}{\xi_{F}}=\frac{1}{\xi_{F}}\left(\kappa \sqrt{\frac{1}{\tau}+\rho^{2}}-\lambda \rho-1\right)
$$

the squared scaling variable lin , linked to the minimum energy $\epsilon_{0}$ required to a nucleon in order to respond to the probe. In the above $\tau=\kappa^{2}-\lambda^{2}$ and

$$
\rho=1+\frac{1}{4 \tau}\left(\mu^{2}-1\right) \quad \text { with } \quad \mu=m_{\Delta} / m_{N}
$$

is an inelasticity parameter which reduces to unity in the nucleonic sector, thus allowing for a compact treatment of the quasi-elastic and $\Delta$ peaks, providing 
the $\Delta$ is treated as a stable particle: the resonance decay can be subsectently taken into account by introducing a finite, energy-dependent, width 닌.5.

The functions $U$ and $\widetilde{U}$ in (3,, A $^{2}$ depend upon the specific electron-nucleon process of interest, although they are not simply given by single nucleons form factors, since in a relativistic model the nucleonic and the many-body content of the problem cannot be factorized. They read

$$
\begin{aligned}
& U^{L}(\kappa, \lambda)=\frac{\kappa^{2}}{\tau}\left[\left(1+\tau \rho^{2}\right) w_{2}(\tau)-w_{1}(\tau)+w_{2}(\tau) \mathcal{D}^{L}(\kappa, \lambda)\right] \\
& U^{T}(\kappa, \lambda)=2 w_{1}(\tau)+w_{2}(\tau) \mathcal{D}^{T}(\kappa, \lambda) \\
& \widetilde{U}^{L}(\kappa, \lambda)=\frac{\kappa^{2}}{\tau}\left[\left(1+\tau \rho^{2}\right) \widetilde{w}_{2}(\tau)-\widetilde{w}_{1}(\tau)+\widetilde{w}_{2}(\tau) \mathcal{D}^{L}(\kappa, \lambda)\right] \\
& \widetilde{U}^{T}(\kappa, \lambda)=2 \widetilde{w}_{1}(\tau)+\widetilde{w}_{2}(\tau) \mathcal{D}^{T}(\kappa, \lambda) \\
& \widetilde{U}^{T^{\prime}}(\kappa, \lambda)=2 \sqrt{\tau\left(\tau \rho^{2}+1\right)} \widetilde{w}_{3}(\tau)\left[1+\mathcal{D}^{T^{\prime}}(\kappa, \lambda)\right],
\end{aligned}
$$

where the functions $w_{i}, \widetilde{w}_{i}$ contain the single nucleon electromagnetic and weak form factors respectively (see Refs. 2 and 5 for their expressions). The functions $\mathcal{D}^{L, T, T^{\prime}}$ arise from the relativistic kinematics and read

$$
\begin{aligned}
& \mathcal{D}^{L, T}(\kappa, \tau)=\frac{1}{\epsilon_{F}-\epsilon_{0}} \int_{\epsilon_{0}}^{\epsilon_{F}} \eta_{T}^{2}(\epsilon) d \epsilon \\
& =\frac{\tau}{\kappa^{2}}\left[(\lambda \rho+1)^{2}+(\lambda \rho+1)\left(1+\psi^{2}\right) \xi_{F}+\frac{1}{3}\left(1+\psi^{2}+\psi^{4}\right) \xi_{F}^{2}\right]-\left(1+\tau \rho^{2}\right) \\
& \mathcal{D}^{T^{\prime}}(\kappa, \tau)=\frac{1}{\epsilon_{F}-\epsilon_{0}} \int_{\epsilon_{0}}^{\epsilon_{F}}\left(\sqrt{1+\frac{\eta_{T}^{2}(\epsilon)}{1+\tau \rho^{2}}}-1\right) d \epsilon \\
& =\frac{1}{\kappa} \sqrt{\frac{\tau}{1+\tau \rho^{2}}}\left[1+\xi_{F}\left(1+\psi^{2}\right)+\lambda \rho\right]-1
\end{aligned}
$$

The above expressions show that $\mathcal{D}^{L}$ and $\mathcal{D}^{T}$ just correspond to the mean square value of the transverse momentum

$$
\eta_{T}(\epsilon)=\sqrt{\frac{\tau}{\kappa^{2}}(\epsilon+\lambda \rho)^{2}-1-\tau \rho^{2}}
$$

of the nucleon, whereas $\mathcal{D}^{T^{\prime}}$ is related to the transverse kinetic energy of the nucleon when $\tau$ is small. It is worth noticing that the $\mathcal{D}$ functions are quadratic in the Fermi momentum and vanish in non-relativistic models. 
The relativistic response functions thus obtained are sensibly different from the non-relativistic ones for momentum transfer higher than about 1 $\mathrm{GeV} / \mathrm{c}$, the differcence obviously growing with $q$.

As an illustration, we focuss on the $\Delta$ region, where the transverse Fermi motion gives rise to a longitudinal response function, even for a spherical (M1) $\Delta$, as predicted by the constituent quark model. This effect, first pointed out in Ref. 6 , is negligible at low $q$ and $k_{F}$, but becomes significant in heavy nuclei and for high momentum transfer. Moreover, if one considers the full $N \rightarrow \Delta$

vertex $\mathrm{G}$, that includes $\mathrm{M} 1, \mathrm{E} 2$ and $\mathrm{C} 2$ amplitudes, the longitudinal response in the $\Delta$ region turns out to be

$$
R_{\Delta}^{L}(\kappa, \lambda) \propto G_{C, \Delta}^{2}(\tau)+\frac{4 \mu^{2}\left[G_{M, \Delta}^{2}(\tau)+3 G_{E, \Delta}^{2}(\tau)\right]+G_{C, \Delta}^{2}(\tau)}{\tau\left(1+\tau \rho^{2}\right)} \mathcal{D}^{L}(\kappa, \tau),
$$

thus arising from both the Coulomb transition form factor and from the manybody relativistic effect contained in $\mathcal{D}$. This effect, evaluated in Ref. 4 in the RFG model, yields a $\Delta$ longitudinal response which is about $15 \%$ of the corresponding quasi-elastic one at $\mathrm{q}=1 \mathrm{GeV} / \mathrm{c}$ in absence of Coulomb form factor. The latter is shown to further enhance this ratio of almost a factor 2, thus showing the strong sensitivity of this response to the deformation of the $\Delta$ resonance.

To go beyond the free Fermi gas, the impact of the N-N interaction on the respgnse functions should be calculated in the same relativistic framework. In Ref. $B$ the effect of nuclear correlations on $R_{\Delta}^{L}$ is included in the Boson Loop Expansion framework, which sums up an infinite class of particle-hole, $\Delta$-hole, $2 \mathrm{p}-2 \mathrm{~h}$ and $3 \mathrm{p}-3 \mathrm{~h}$ diagrams: the results indicate a substantial enhancement of the response at $q \simeq 500 \mathrm{MeV} / \mathrm{c}$. Unfortunately the calculation could not be applied at higher momentum transfers, since it is non-relativistic. Although exact relativistic calculations can in principle be performed, we illustrate in the next Section a new non-relativistic expansion of the nuclear currents, first suggested in Ref. 9, which provides an extremely good approximation to the exact result for any value of the energy and momentum transfers and allows for the inclusion of relativistic effects in non-relativistic calculations through simple kinematical factors.

\section{A new non-relativistic expansion}

The method treats asymmetrically the nucleons in initial and final states, expanding the single nucleon current in powers of the parameter $\eta=p / m_{N}$, where $p$ is the three-momentum of the nucleon inside the Fermi sphere, and

paper: submitted to World Scientific on November 5, 2018 
truncating the expansion at linear order. Since $\eta \leq \eta_{F}, \eta_{F}$ being tipycally about $1 / 4$, this turns out to be an excellent approximation, which, compared to the traditional non-relativistic expansions, has the advantage of retaining the full dependence on $\kappa$ and $\lambda$. The same procedure can also be applied to 2 -body currents, like meson-exchange 10 and correlation currents 11 . The "relativized" currents so obtained can be implemented together with relativistic kinematics in standard non-relativistic models of one-particle emission near the quasi-elastic and $\Delta$ peaks.

To illustrate the method, let us discuss in some details the single-nucleon on-shell electromagnetic current operator

$$
J^{\mu}\left(P^{\prime} s^{\prime} ; P s\right)=\bar{u}\left(\mathbf{p}^{\prime}, s^{\prime}\right)\left[F_{1} \gamma^{\mu}+\frac{i}{2 m_{N}} F_{2} \sigma^{\mu \nu} Q_{\nu}\right] u(\mathbf{p}, s) .
$$

By expanding the Dirac spinors in powers of the bound nucleon momentum $\boldsymbol{\eta}=\mathbf{p} / m_{N}$ one gets (omitting the spinors $\chi_{s}, \chi_{s^{\prime}}^{\dagger}$ )

$$
\begin{aligned}
J^{0} & \simeq \frac{\kappa}{\sqrt{\tau}} G_{E}+\frac{i}{\sqrt{1+\tau}}\left(G_{M}-\frac{G_{E}}{2}\right)(\boldsymbol{\kappa} \times \boldsymbol{\eta}) \cdot \boldsymbol{\sigma} \\
\boldsymbol{J}^{\perp} & \simeq \frac{1}{\sqrt{1+\tau}}\left\{i G_{M}(\boldsymbol{\sigma} \times \boldsymbol{\kappa})+\left(G_{E}+\frac{\tau}{2} G_{M}\right)\left(\boldsymbol{\eta}-\frac{\boldsymbol{\kappa} \cdot \boldsymbol{\eta}}{\kappa^{2}} \boldsymbol{\kappa}\right)\right. \\
& \left.-\frac{i G_{M}}{1+\tau}(\boldsymbol{\sigma} \times \boldsymbol{\kappa}) \boldsymbol{\kappa} \cdot \boldsymbol{\eta}+\frac{i G_{M}}{2(1+\tau)}(\boldsymbol{\eta} \times \boldsymbol{\kappa}) \boldsymbol{\sigma} \cdot \boldsymbol{\kappa}\right\}
\end{aligned}
$$

for the time and space transverse components, respectively, the longitudinal current being trivially linked to $J^{0}$ via current conservation. By comparing these expressions with the traditional non-relativistic ones

$$
\begin{aligned}
J^{0}{ }_{\text {nonrel }} & =G_{E} \\
\boldsymbol{J}_{\text {nonrel }}^{\perp} & =i G_{M}(\boldsymbol{\sigma} \times \boldsymbol{\kappa})+G_{E}\left(\boldsymbol{\eta}-\frac{\boldsymbol{\kappa} \cdot \boldsymbol{\eta}}{\kappa^{2}} \boldsymbol{\kappa}\right)
\end{aligned}
$$

one notices that, besides the extra terms linear in $\eta$, the kinematical factor $\kappa / \sqrt{\tau}>1$ is present in the time component, corresponding to an enhancement of the charge response function, whereas the factor $1 /(1+\tau)<1$ in $\boldsymbol{J}^{\perp}$ induces a reduction of the transverse response. These effects are directly linked to the relativistic phenomena of length contraction and time dilation.

Although technically more cumbersome because of the spin structure of the $\Delta$, the same procedure can be applied to the $N \rightarrow \Delta$ transition current. If, for simplicity, we restrict our analysis to M1 transistions, the $\eta$-expansion of the current 0 

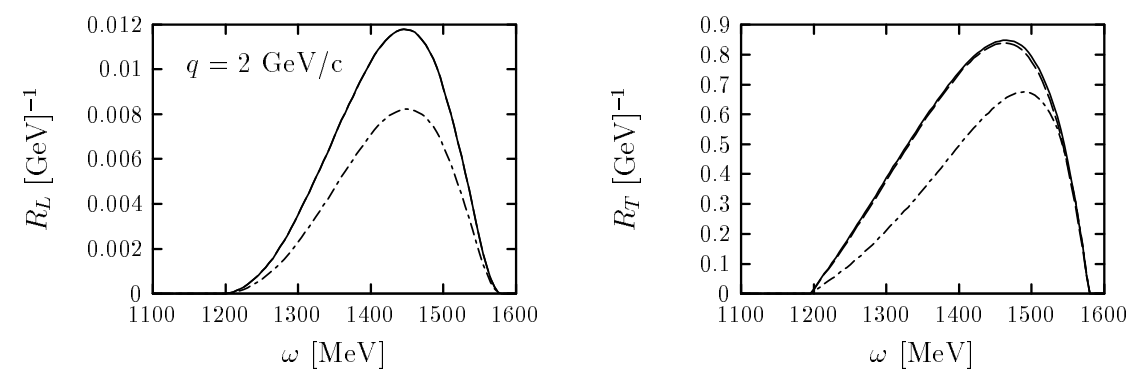

Figure 1. Electromagnetic responses in the $\Delta$-peak at $q=2 \mathrm{GeV} / \mathrm{c}$ for ${ }^{12} \mathrm{C}$ with $k_{F}=225$ $\mathrm{MeV} / \mathrm{c}$, without a $\Delta$-width. Solid: exact results within the RFG model; dashed: new expansion of the electromagnetic current to first order in $\eta$; dot-dashed: traditional nonrelativistic current. We use relativistic kinematics in all cases.

$$
J_{N \rightarrow \Delta}^{\mu}\left(P_{\Delta} s_{\Delta} ; P s\right)=\bar{u}_{\Delta}^{\beta}\left(P_{\Delta}, s_{\Delta}\right) \frac{G}{2 m_{N}^{2}} \epsilon^{\beta \mu \alpha \gamma}\left(P_{\alpha}+P_{\Delta \alpha}\right) Q_{\gamma} u(P, s),
$$

where $u_{\Delta}^{\beta}\left(P_{\Delta}, s_{\Delta}\right)$ is the Rarita-Schwinger spinor describing a spin-3/2 particle and $G$ is proportional to the magnetic transition form factor $G_{M, \Delta}$, yields

$$
\begin{aligned}
J_{N \rightarrow \Delta}^{0} & =-2 G \sqrt{1+\tau^{\prime}} \mathbf{S}^{\dagger} \cdot(\boldsymbol{\eta} \times \boldsymbol{\kappa})+O\left(\eta^{2}\right) \\
\boldsymbol{J}_{N \rightarrow \Delta} & =2 G \sqrt{1+\tau^{\prime}}\left(\mathbf{S}^{\dagger} \times \boldsymbol{\kappa}\right)+O(\eta),
\end{aligned}
$$

where $\tau^{\prime}=\left[4 \tau+(\mu-1)^{2}\right] /(4 \mu)$ and $\mathbf{S}$ is the usual $1 / 2 \rightarrow 3 / 2$ spin transition operator.

The quality of the new approximation to the relativistic, magnetic $\Delta$ current is shown in fig. 1, where the exact RFG longitudinal and transverse responses obtained using magnetic and electric form factors are displayed with solid lines. In addition we show with dashed lines the responses computed in the non-relativistic Fermi gas model with relativistic kinematics and the new currents in Eqs. (22, 23). For comparison we also show with dot-dashed lines results for the non-relativistic Fermi gas model and relativistic kinematics, but using the traditional non-relativistic current. The improvement of the description of the relativistic results using our currents is clear from this figure - the solid and dashed lines almost coincide. This proves that our expansion to order $O(\eta)$ is precise enough to describe the $\Delta$ excitation in nuclei with negligible error for high momentum transfers. 


\section{Meson Exchange Currents}

We now consider the Meson-Exchange-Currents (MEC) associated to pion exchange, which have to be taken into account in order to preserve current conservation. We have applied the above non-relativistic expansion to the particle-hole matrix elements of the so-called pion-in-flight and seagull currents, corresponding to $\gamma \pi \pi$ and $\gamma \pi N$ vertices respectively, and compared the results with the exact relativistic calculation and with the "traditional" non-relativistic one, where $q$ and $\omega$ are not treated exactly 12 .

In Fig. 2 we show, for sake of illustration, the p-h matrix element of a typical component of the pion-in-flight current at $q=2 \mathrm{GeV} / \mathrm{c}$ in the allowed spin channels (spin diagonal in the left panel and spin-flip in the right panel); similar results hold for the other components and for the seagull current, as shown in Ref. 10. Again our approximation is in fact indistinguishable from the exact calculation.

Notably the final expressions for the "relativized" currents $(\mathrm{R})$ is linked to the traditional non-relativistic (TNR) ones through simple kinematical factors. For the pion-in-flight this is a global reduction factor

$$
J_{P}^{\mu, R}=\frac{1}{\sqrt{1+\tau}} J_{P}^{\mu, T N R}
$$

and the same relation holds for the time component of the seagull current, whereas for its space component we get

$$
\boldsymbol{J}_{S}^{R}=\frac{1}{\sqrt{1+\tau}} \boldsymbol{J}_{S}^{1, T N R}+\sqrt{1+\tau} \boldsymbol{J}_{S}^{2, T N R},
$$

where $\boldsymbol{J}_{S}^{1}$ and $\boldsymbol{J}_{S}^{2}$ correspond to two different pionic momentum flows (see Ref. 10 for details).

\section{The asymmetry in the $\Delta$-resonance region}

Due to the purely isovector nature of the $\Delta$ resonance, the asymmetry (2) reads in this region

$$
\mathcal{A}_{N-\Delta}=\mathcal{A}_{0}\left\{-\left(1-2 \sin ^{2} \theta_{w}\right)+v_{T^{\prime}} \frac{\widetilde{R}_{V A}^{T^{\prime}}(\kappa, \lambda)}{v_{L} R^{L}(\kappa, \lambda)+v_{T} R^{T}(\kappa, \lambda)}\right\},
$$

$\theta_{w}$ being the Weinberg angle. The above formula clearly shows that if the axial $N \rightarrow \Delta$ response can be neglected then the inelastic asymmetry, normalized 

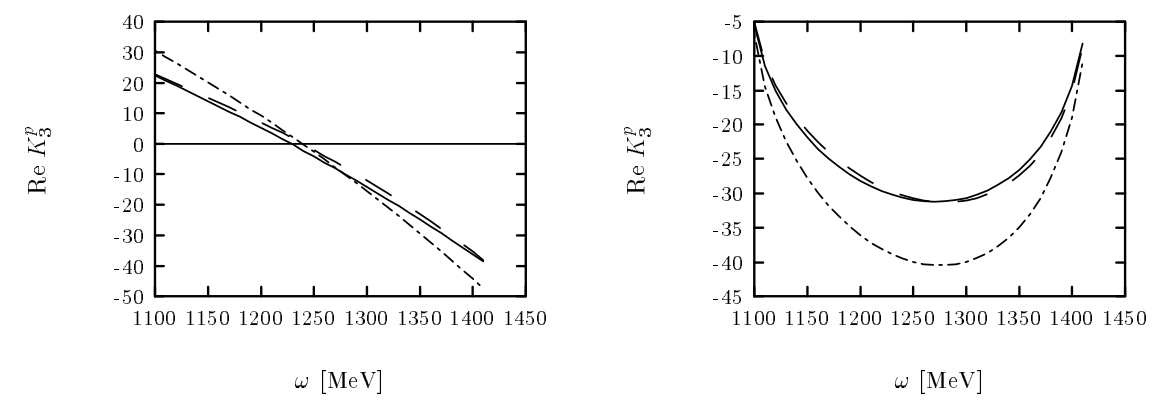

Figure 2. Pion-in-flight $\mathrm{p}-\mathrm{h}$ matrix elemnts (in arbitrary units) for $\mathrm{q}=2 \mathrm{GeV} / 5$. The kinematics for the hole is $|\vec{h}|=175 \mathrm{MeV} / \mathrm{c}$ and the azimuthal angle is $\Phi=0$. The spins are $s_{p}=s_{h}=1 / 2$ in the left panel and $s_{p}=-s_{h}=1 / 2$ in the right panel. Soild: relativistic; dashed: our approximation; dot-dashed: traditional non-relativistic
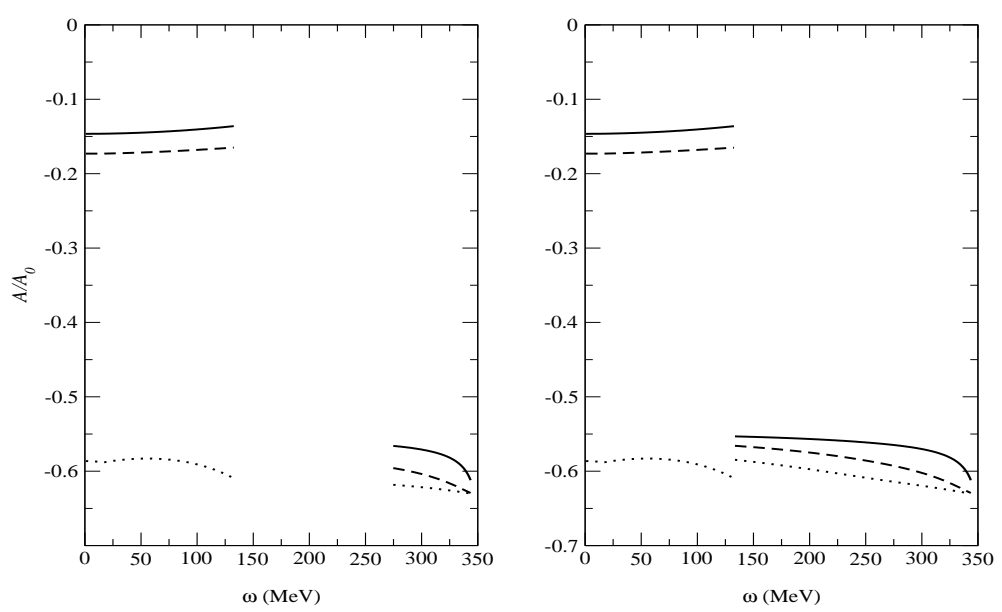

Figure 3. Asymmetry at $\theta=10^{\circ}$ (solid), $\theta=30^{\circ}$ (dashed) and $\theta=150^{\circ}$ (dotted) for $q=350 \mathrm{MeV} / \mathrm{c}$. The $\omega$ range encompasses both the QEP and the $\Delta$ domain. The left and right panels respectively refer to a vanishing width and to a finite decay width $\Gamma(s)$.

to $\mathcal{A}_{0}$ and displayed versus $\lambda$ for fixed $\kappa$, would be flat in the $\Delta$ domain. Hence a departure from flatness would signal the presence of the axial response. In Fig. 3 the ratio $\mathcal{A} / \mathcal{A}_{0}$ is displayed versus $\omega$ at $q=350 \mathrm{MeV} / \mathrm{c}$ and $\theta=10^{0}$, which are, according to the analysis of Ref. 5 , the most favourable kinematical conditions: indeed the asymmetry is increased by about $10 \%$. 
The most notable feature of the $\mathcal{A}$ found relates to the dramatic increase of its magnitude as one makes a transition from the QEP into the $N \rightarrow \Delta$ region for small electron scattering angles. Because of its size this effect should be measurable both at large $(\sim 1 \mathrm{GeV} / \mathrm{c})$ and moderate $(\sim 300-400 \mathrm{MeV} / \mathrm{c})$ momentum transfer. In the former case nuclear interactions are not likely to disrupt the RFG predictions too much. In the latter a modification of the effect could take place, but then this might eventually help to shed light on the nature of the $N N$ and $N \Delta$ forces.

\section{Acknowledgments}

The work presented is the result of collaborations with L. Alvarez-Ruso, J.E. Amaro, P. Amore, J.A. Caballero, R. Cenni, T.W. Donnelly and A. Molinari.

\section{References}

1. T.W. Donnelly, M.J. Musolf, W.M. Alberico, M.B. Barbaro, A. De Pace and A. Molinari, NPA 541, 525 (1992).

2. L. Alvarez-Ruso, M.B. Barbaro, T.W. Donnelly and A. Molinari, nuclth/0007036, in print on PLB.

3. W.M. Alberico, A. Molinari, T.W. Donnelly, E.L. Kronenberg and J.W. Van Orden, PRC 38, 1801 (1988).

4. J.E. Amaro, M.B. Barbaro, J.A. Caballero, T.W. Donnelly and A. Molinari, NPA 657, 161 (1999).

5. P. Amore, R. Cenni, T. W. Donnelly and A. Molinari, nucl-th/0007072, in print on NPA.

6. G. Chanfray, J. Delorme, M. Ericson and A. Molinari, NPA 556, 439 (1993).

7. H.F. Jones and M.D. Scadron, Ann. Phys. 81, 1 (1973).

8. P. Amore, R. Cenni, P. Saracco, Nuovo Cimento A 112, 1015 (1999).

9. S. Jeschonnek and T.W. Donnelly, PRC 57, 2438 (1998).

10. J.E. Amaro, M.B. Barbaro, J.A. Caballero, T.W. Donnelly and A. Molinari, NPA 643, 349 (1998).

11. J.E. Amaro, M.B. Barbaro, J.A. Caballero, T.W. Donnelly and A. Molinari, in preparation.

12. W.M. Alberico, T.W. Donnelly and A. Molinari, NPA 512, 541 (1990).

paper: submitted to World Scientific on November 5, 2018 